\title{
Multiple epiphyseal dysplasia, Al-Gazali type
}

INSERM

\section{Source}

INSERM. (1999). Orphanet: an online rare disease and orphan drug data base. Multiple epiphyseal dysplasia, Al-Gazali type. ORPHA:166024

Multiple epiphyseal dysplasia, Al-Gazali type is a skeletal dysplasia characterized by multiple epiphyseal dysplasia (see this term), macrocephaly and facial dysmorphism. 